DOI 10.1007/s00167-005-0703-0

\author{
Rochelle L. Nicholls \\ Daniel Green \\ Markus S. Kuster
}

\section{Patella intraosseous blood flow disturbance during a medial or lateral arthrotomy in total knee arthroplasty: a laser Doppler flowmetry study}

the medial patella (53\%) compared to the lateral approach $(27 \%)$. A large standard deviation of scores was evident in all cases. Although there was a tendency for LA to disturb the patellar blood flow less, the difference was not significant. It was concluded that neither approach is superior regarding the blood flow preservation to the patella. Hence, a lateral approach might only have an advantage in knee joints that are likely to need a lateral release in combination with an MA-e.g., a valgus deformity or preoperative patella maltracking.

Keywords Regional blood flow . Arthroplasty (knee replacement)

\section{Introduction}

Patella complications are recognized sequelae of total knee arthroplasty (TKA). Anterior knee pain, patella maltracking, osteonecrosis, and patella fractures constitute up to $50 \%$ of post-TKA complications [5-9]. Disruption of blood flow to the patella during surgery may contribute to reduced viability of the bone and patella tendon [14], and is therefore thought to play an important role for patella complications. Several studies have investigated the effects of a medial arthrotomy (MA) with or without a lateral release on patellar blood flow in vitro [3, 15], in vivo [4, 21, 25], and in animal models [23]. In particular, an MA in combination with a lateral release has been shown to compromise the patellar blood flow, and has been associated with an increased incidence of osteonecrosis [11] and patella fractures [24]. Since the prevalence for a lateral release is high in valgus knees, a lateral approach has been suggested in these cases [16]. The lateral approach in combination with a tubercle osteotomy has even been suggested as a standard approach for varus knees to improve patella tracking, and to better preserve blood supply [1]. However, the effect of a lateral arthrotomy (LA) on blood flow to the patella and associated soft tissues has not yet been investigated in vitro or in vivo. It is therefore the purpose of the present study to compare patellar blood flow changes during an LA or an MA in total knee replacement using laser Doppler flowmetry (LDF) intraoperatively. 


\section{Materials and methods}

Patients

Informed consent was obtained from 16 osteoarthritis patients awaiting primary TKA surgery (4 male, 12 female patients; mean age $75.3 \pm 5.7$ years). One surgeon (MSK) performed all procedures. Each patient was randomly assigned to the MA $(n=8)$ or LA treatment group $(n=8)$, irrespective of preoperative varus or valgus alignment.

\section{Blood flow measurement}

Laser Doppler flowmetry provides continuous real-time monitoring of vascular perfusion in vivo. An optical probe placed on the soft tissue surface or into cancellous bone carries a beam of monochrome light generated by a low-power helium-neon laser. The laser light penetrates the tissue and is repeatedly refracted and reflected (scattered) by the tissue. Light that hits moving cells results in a Doppler shift (change in wavelength) proportional to the number and velocity of cells. Optical fibers in the probe convey back-scattered light to photodetectors which convert the light to an electrical signal proportional to cell motion (in perfusion units, $\mathrm{mV}$ ). LDF has previously been shown to provide a stable, reproducible method with which to measure relative flow change in vivo [20, 29].

LDF was undertaken using the PeriMed System 5000 unit (PeriMed, Stockholm, Sweden) and two PeriMed 403 probes (length $40 \mathrm{~mm}$, diameter $1 \mathrm{~mm}$ ). The system was interfaced with a PC notebook computer (Toshiba Satellite-A10) via a National Instruments BNC-2110 connector block and 6024E 12-bit data acquisition card (National Instruments, Austin, TX, USA).

The body temperature, systemic blood pressure, and heart rate of each patient were monitored continuously during the procedure. After the skin incision, baseline LDF measures were made without tourniquet, using two probes inserted into holes $(1.5 \mathrm{~mm} \times 5 \mathrm{~mm})$ drilled $10 \mathrm{~mm}$ either side of the midline of the anterior patella (Fig. 1). A further measurement was then made in the center of the patella tendon by suturing a customized probe support to the ligament surface (Fig. 2). This addition to the usual surgical procedure was undertaken to avoid the large variation in signal which occurs when the probe is held in the hand [19]. After arthrotomy, measurements were repeated. The surgeon was blinded to the results of all LDF procedures. All readings were taken with the leg in $90^{\circ}$ of flexion. As a means of validating the method, flow measures were repeated after inflating the femoral tourniquet.

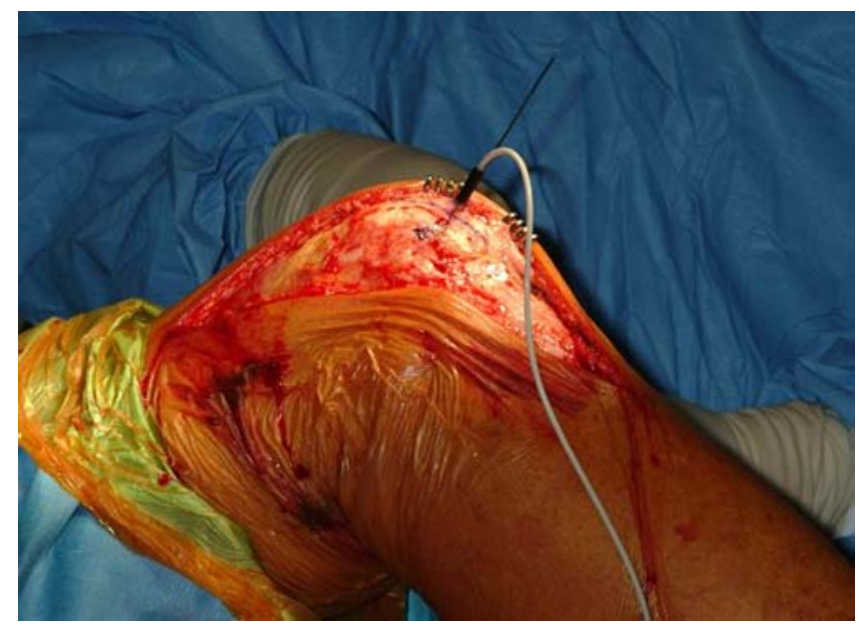

Fig. 1 LDF probe in situ in the lateral patella. Also illustrated is a $\mathrm{K}$-wire inserted in the $1.5-\mathrm{mm}$ hole drilled for measurements in the medial patella

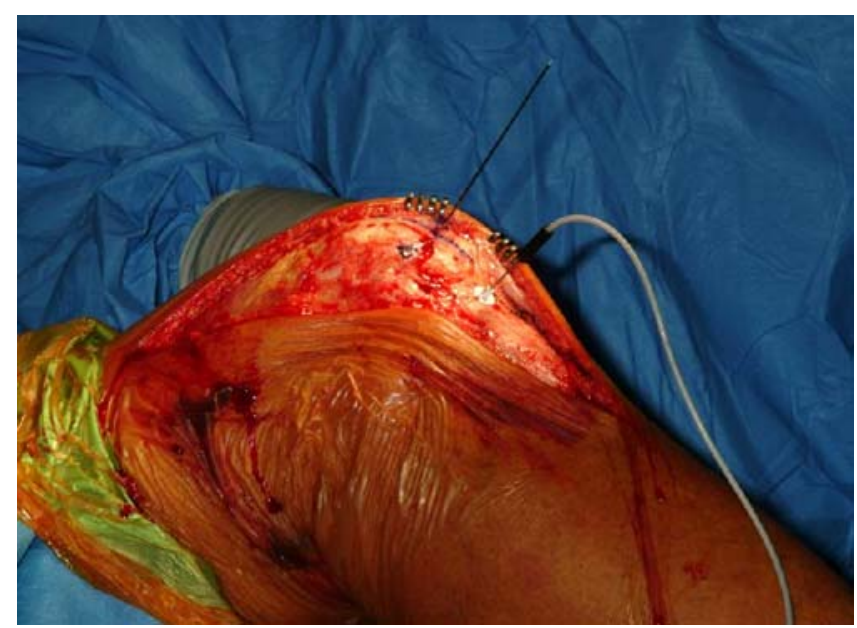

Fig. 2 LDF probe in situ in the patella ligament. The customized probe holder is shown sutured to the tissue

\section{Data reduction}

During LDF procedures, data were sampled at $50 \mathrm{~Hz}$ for $60 \mathrm{~s}$ using a program written in LabView $7.0(\mathrm{Na}-$ tional Instruments, Austin, TX, USA). A customized processing routine in Matlab 6.5 (MathWorks, Natick, MA, USA) was used to remove the 40-s stabilizing period from the signal and apply a low-pass Butterworth filter (cut-off frequency of $5 \mathrm{~Hz}$ ) to the remaining data. The flow was reported as the mean of this filtered signal $(\mathrm{mV})$. A Fourier transform was performed to check the data for aberrant frequencies, and to illustrate the effect of the patient's heart rate on LDF signal. All data were normalized to the baseline value, and this was assigned a value of $100 \%$ to facilitate comparison of flow changes between MA and LA conditions. 
Statistical analysis

The effect on patella and patella ligament vascular perfusion of medial or lateral arthrotomy was assessed using the Mann-Whitney $U$ nonparametric test for equality of variables sampled from a non-normal distribution [31]. The alpha-level of significance was set at $P<0.05$.

\section{Results}

The characteristics of treatment groups after randomization of patients into MA and LA groups are given in Table 1. The groups are comparable in patient age, body mass index (BMI), preoperative knee alignment, and flexion range. There was also an equal number of patients in each group with symptoms of hypertension or peripheral vascular disease $(n=4)$.

\section{Medial versus lateral arthrotomy}

The mean changes in blood flow following arthrotomy are given in Table 2. Flow in the lateral and medial patella was substantially reduced for both MA and LA compared to the baseline readings (Fig. 3). Although the medial patellar blood flow showed a larger blood flow reduction after MA (53\%) compared to LA (27\%), this difference was not statistically significant $(P=0.30)$. Patella tendon flow was only reduced in two cases each for MA and LA, and this flow reduction was not significant $(P=0.20)$. A large variability of flow changes across patients was noted. While some patients showed little if any blood flow change after arthrotomy, greater reductions (up to $85 \%$ ) occurred in other cases.

\section{LDF validity}

The sensitivity to flow of LDF was tested by placing the probe in the medial and lateral patella after inflating the femoral tourniquet. The flow reduced to zero in both cases, indicating that the measurements were not obscured by external artifact. Results of the Fourier transform revealed that the main frequency of the signal was the pulse rate of the patient (Fig. 4). As was already shown by other authors [28], the probes are sensitive to movements during data acquisition. Measurements made with and without the customized probe holder sutured to the patella ligament showed a large variation in signal (Fig. 5).

\section{Discussion}

The blood supply to the patella has been extensively investigated $[2,12,17,26,27,30]$. The main supply occurs through an anastomotic ring consisting of the anterior tibial recurrent artery and five genicular arteries, which penetrate anteriorly through the bone through vascular foramina. Three main arteries originate from the medial side and three from the lateral side. An additional source of blood supply has also been shown to occur through the patella tendon and quadriceps tendon [2]. Hughes et al. (1988) used LDF through a single $5 \mathrm{~mm}$ hole in the center of the patella to show a blood flow reduction of $41 \%$ after an MA, which compares well with our findings. The present study was the first to investigate the effects of an LA on the blood supply, and found no significant difference between the MA and the LA, indicating that both the lateral and the medial arteries evenly contribute to the patella blood supply.

The present study also investigated the effects of an arthrotomy on patella tendon blood flow, and did not find a significant blood flow reduction for both the LA and the MA. We consider preservation of the fat pad important for the vitality of the patella tendon. Hence, the fat pad was preserved during surgery in all cases, which might well be the reason for this finding. The fat pad has been shown to have an anastomotic network from the lateral and medial side, and contribute to the blood supply of the patella tendon [26]. Branches of the inferior genicular and recurrent anterior tibial arteries provide blood flow from the lateral aspect of the joint, with medial supply from branches of the inferior medial genicular artery [18, 26]. Hughes et al. [13] measured the bony blood flow after fat pad resection and found no effect. Further research is recommended to determine if resection of the fat pad would interfere more with the blood supply of the patella tendon than with the patella bone.

Table 1 Patient characteristics for MA and LA treatment groups

\begin{tabular}{llllllll}
\hline $\begin{array}{l}\text { Treatment } \\
\text { group }\end{array}$ & $\begin{array}{l}\text { Mean age } \\
\text { (years) }\end{array}$ & $\begin{array}{l}\text { Varus-aligned } \\
\text { knees }(n)\end{array}$ & $\begin{array}{l}\text { Valgus-aligned } \\
\text { knees }(n)\end{array}$ & $\begin{array}{l}\text { Mean pre-operative } \\
\text { flexion range }\left(^{\circ}\right)\end{array}$ & $\begin{array}{l}\text { Patients with } \\
\text { extension } \\
\text { deficient }>5^{\circ}(n)\end{array}$ & $\begin{array}{l}\text { Mean } \\
\text { BMI }\end{array}$ & $\begin{array}{l}\text { Patients with } \\
\text { hypertensive or peripheral } \\
\text { vascular disease }(n)\end{array}$ \\
\hline LA $(n=8)$ & $76.1 \pm 5.7$ & 4 & 4 & $116 \pm 11.5$ & 1 & $30.4 \pm 4.9$ & 4 \\
MA $(n=8)$ & $74.9 \pm 6.8$ & 3 & 5 & $108 \pm 23.1$ & 2 & $32.2 \pm 4.8$ & 4 \\
\hline
\end{tabular}


Table 2 Changes in arterial flow (\%) following medial or lateral arthrotomy during TKA

\begin{tabular}{lll}
\hline Surgical approach & Lateral patella $(\%)$ & Medial patella $(\%)$ \\
\hline MA & $19 \pm 41$ & $53 \pm 38$ \\
LA & $22 \pm 46$ & $27 \pm 42$ \\
$P(\alpha=0.05)$ & 0.337 & 0.172 \\
\hline
\end{tabular}

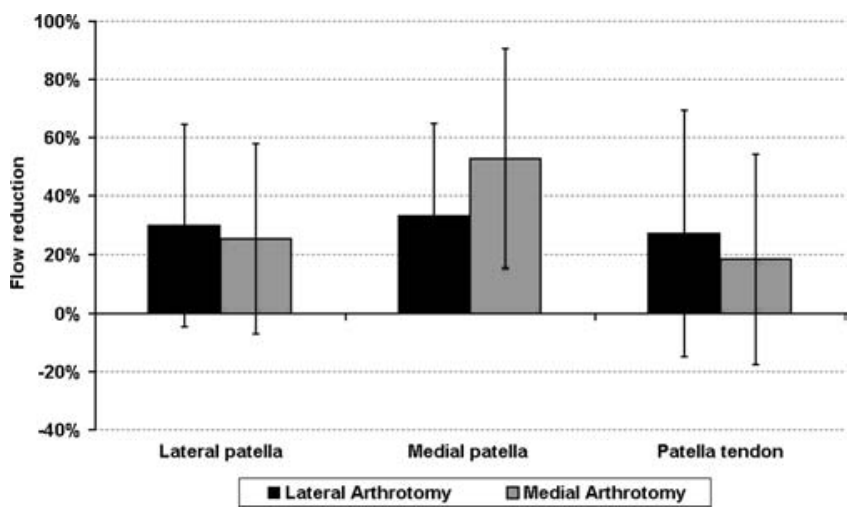

Fig. 3 Mean change in genicular flow (\%) after arthrotomy in TKA

The fact that some patellae showed a marked change in perfusion after an MA or an LA, while others showed little change necessitates further discussion. A potential explanation for this large variability may be the localized area of the LDF readings in the bone. Despite the use of two measurement sites, LDF provides only a localized measure of vascular perfusion. A 1-mm diameter probe can only survey an area of $1 \mathrm{~mm}^{3}$ around the tip, with a maximum penetration of $3.5 \mathrm{~mm}$ into cancellous bone [22]. Hence the measurements made in this study are site-specific and might not be extrapolated to the whole patella. However, our findings are supported by cadaver and animal studies. Ogata et al. [23] used the hydrogen washout technique to demonstrate a $35 \%$ decrease in patellar blood flow after an MA in monkeys, which is in keeping with the present findings.

Another explanation for the large variability might be anatomical variations and osteoarthritic changes. In some cases, additional blood flow to the patella can be provided through the quadriceps and patella tendon as suggested by some authors [2], while other individuals may depend more on the genicular arteries. Furthermore, degeneration of patellar articular cartilage has been shown to correlate with destruction of arterial anastomoses and disruption of patellar blood supply [2, 27]. Hence the intrinsic blood supply to the osteoarthritic patella might already be jeopardized prior to surgery. TKA surgery would have a much greater impact on the blood supply in patellas where arterial anastomoses were destroyed by cartilage degeneration. Scintigraphic studies have shown similar results [25]. In one study [21], only 6 of 46 knee joints after TKA with a medial parapatellar approach showed a cold postoperative bone scan, indicating individual anatomical variability in blood flow. A prospective study [10] used bone scans in 30 patients after TKA without patellar resurfacing and without lateral release to show a transient patellar ischemia in about $14 \%$ of cases, with those patients also reporting ongoing anterior knee pain.

It can be concluded from the present study that the use of LA has no superior flow-preserving effects, contrary to the suggestions of previous authors [1]. However, a lateral approach incorporates the lateral release into the arthrotomy, and a bilateral incision of the capsule is never necessary to improve patella tracking. After an MA, a compromise must sometimes be made between blood supply and patella tracking. McMahon et al. [21] demonstrated a higher incidence of patella
Fig. 4 FFT chart illustrating the frequencies associated with patient heart rate as a major variant in LDF signal

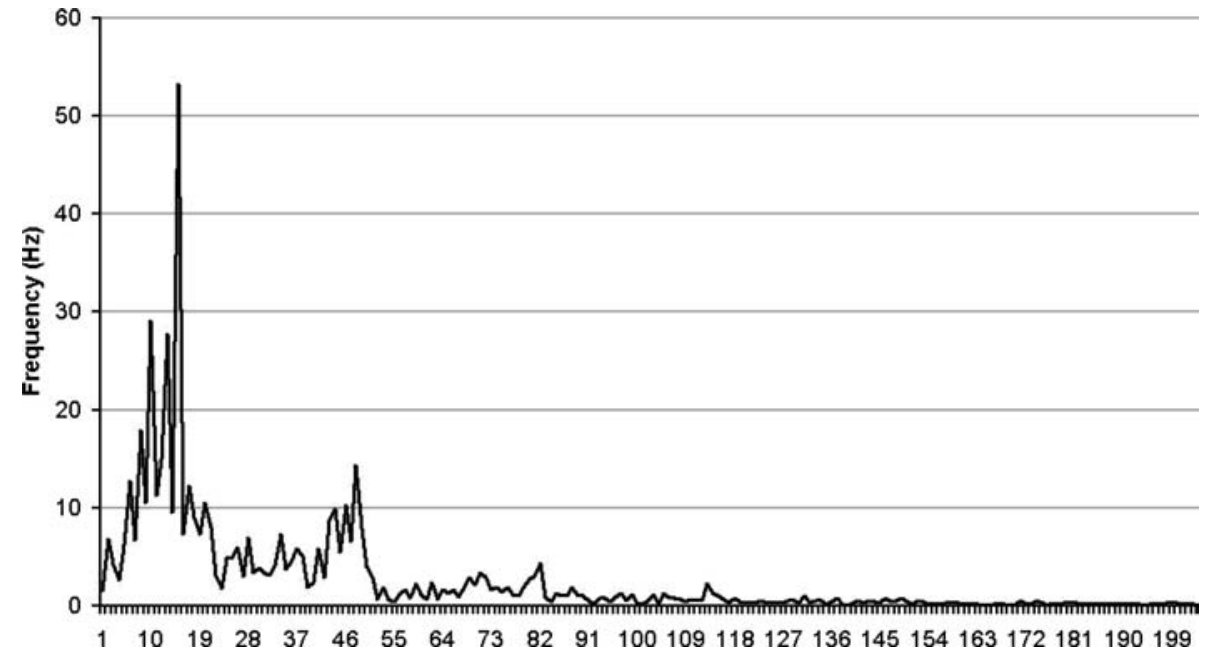


Fig. 5 Probe stabilization technique affects the quality of data obtained from LDF procedures, as illustrated from measurements taken in the patella ligament

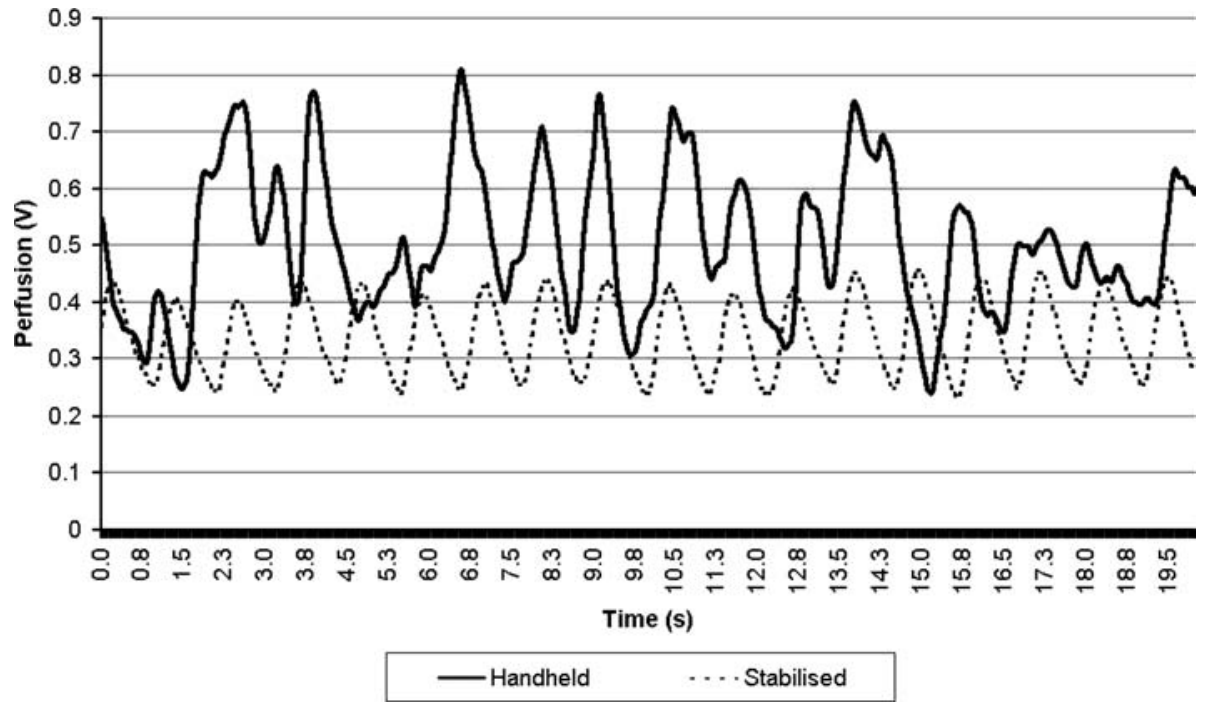

vascular compromise when a lateral release was performed in combination with a medial parapatellar approach. Ritter et al. [24] showed in a large study of 1,205 TKAs that a lateral release in combination with an MA showed significantly more patella fractures, while patella radiolucency was more common in knees without a lateral release.

To the best of our knowledge, this is the first study to compare the blood supply disturbance of the medial to the lateral approach in TKA. Despite extensive measures undertaken to ensure the reliability and validity of measurements, there are several limitations of LDF which must be considered when interpreting the results, including that LDF is a measure of relative (not absolute) flow, and that the localization of mea- surements prevents extrapolation beyond the measurement site. It can be concluded neither approach is superior regarding the blood flow preservation to the patella. However, MA might need a lateral retinacular release in cases where the patella tracking is not optimal. This additional disturbance of the patella blood supply could jeopardize the vitality of the patella. LA never needs an additional retinacular release to improve patella tracking. Hence, LA might be superior to the MA in knee joints that are likely to need a lateral release, such as preoperative valgus deformity or patella maltracking.

Acknowledgements The authors wish to acknowledge the Western Australian Department of Health for supporting the study.

\section{References}

1. Arnold MP, Friederich NF, Widmer H, Muller W (1999) Lateral approach to the knee combined with an osteotomy of the tibial tuberosity. Its use for total knee replacement. Orthop Traumatol 20(3):212-220

2. Bjorkstrom S, Goldie IF (1980) A study of the arterial supply of the patella in the normal state, in chondromalacia patellae and in osteoarthrosis. Acta Orthop Scand 51:63-70

3. Bonutti PM, Miller BG, Cremens MJ (1998) Intraosseous patellar blood supply after medial parapatellar arthrotomy. Clin Orthop Relat Res 352:202-214
4. Brick GW, Scott RD (1989) Blood supply to the patella: significance in total knee arthroplasty. J Arthroplasty 4(Suppl):S75-S79

5. Daum WJ, Simmons DJ, Calhoun JH, Benedetto AR (1988) Regional alterations in long bone produced by internal fixation devices. Part I. 85Sr clearance. J Orthop Trauma 2(3):241-244

6. Daum WJ, Simmons DJ, Chang S, Lehman RC, Webster D (1985) Effect of fixation devices on radiostrontium clearance in the intact canine femur. Clin Orthop Relat Res 194:306-312

7. El-Maraghy AW, Kumeniuk B, Anderson GI, Schemitsch EH, Richards RR (1999) Femoral bone blood flow after reaming and intramedullary canal preparation. A canine study using laser Doppler flowmetry. J Arthroplasty 14(2):220-226
8. El-Maraghy AW, Schemitsch EH, Waddell JP (1999) Greater trochanteric blood flow during total hip arthroplasty using a posterior approach. Clin Orthop Relat Res 363:151-157

9. Field JR, Lord P, Maaripuu E, SumnerSmith G (1999) Semi-quantitiative assessment of tibial blood flow and distribution in response to surgical intervention using first pass radionuclide angiography and intravascular vital dye. Injury 30:681-688 
10. Gelfer Y, Pinkas L, Horne T, Halperin N, Alk D, Robinson D (2003) Symptomatic transient patella ischemia following total knee replacement as detected by scintigraphy. A prospective, randomized, double-blind study comparing the mid-vastus to the medial para-patella approach. Knee 10:341345

11. Holtby RM, Grosso P (1996) Osteonecrosis and resorption of the patella after total knee replacement: a case report. Clin Orthop Relat Res 328:155-158

12. Howard PE, Wilson JW, Robbins TA, Ribble GA (1986) Normal blood supply of the canine patella. Am J Vet Res 47(2):401-403

13. Hughes SS, Cammarata A, Steinmann SP, Pellegrini VDJ (1998) Effect of standard total knee arthroplasty surgical dissection on human patellar blood flow in vivo: an investigation using laser Doppler flowmetry. J South Orthop Assoc 7(3): 198-204

14. Hughes SS, Cammarata A, Steinmann SP, Pellegrini VDJ (1998) Effect of standard total knee arthroscopic surgical dissection on human patellar blood flow in vitro: an investigation using laser Doppler flowmetry. J South Orthop Assoc 7(5): 198-204

15. Kayler DE, Lyttle D (1988) Surgical interruption of patellar blood supply by total knee arthroplasty. Clin Orthop Relat Res 229:221-227

16. Keblish P (1985) Valgus deformity in total knee replacement (TKR): the lateral retinacular approach. Orthop Trans 9:28
17. Kirschner MH, Mench J, Nerlich A, Walser R, Buhren V, Hofmann GO (1997) The arterial blood supply of the human patella. Its clinical importance for the operating technique in vascularized knee joint transplantations. Surg Radiol Anat 19:345-351

18. Kohn D, Deiler S, Rudert M (1995) Arterial blood supply of the intrapatellar fat pad. Anatomy and clinical consequences. Arch Orthop Trauma Surg 114:72-75

19. Kowalski MJ, Schemitsch EH, Kregor PJ, Senft D, Swionkowski MF (1996) Effect of periosteal stripping on cortical bone perfusion: a laser Doppler study in sheep. Calcif Tissue Int 59:24-26

20. Lausten GS, Kiaer T, Dahl B (1993) Laser Doppler flowmetry for estimation of bone blood flow: studies of reproducibility and correlation with microsphere technique. J Orthop Res 11:573-580

21. McMahon MS, Scuderi GR, Glashow JL, Scharf SC, Meltzer LP, Scott WN (1990) Scintigraphic determination of patellar viability after excision of intrapatellar fat pad and/or lateral retinacular release in total knee arthroplasty. Clin Orthop Relat Res 260:10-16

22. Notzli HP, Swiontkowski MF, Thaxter ST, Carpenter GKI, Wyatt R (1989) Laser Doppler flowmetry for bone blood flow measurements: helium-neon laser light attenuation and depth of perfusion assessment. J Orthop Res 7:413-424

23. Ogata K, Shively RA, Shoenecker PL, Chang S (1987) Effects of standard surgical procedures on the patellar blood flow in monkeys. Clin Orthop Relat Res 215:254-259
24. Ritter MA, Herbst SA, Keating EM, Faris PM, Meding JB (1996) Patellofemoral complications following total knee arthroplasty: effect of a lateral release and sacrifice of the superior lateral geniculate artery. J Arthroplasty 11(4):368-372

25. Scuderi GR, Scharf SC, Meltzer LP, Nisonson B, Scott WN (1987) Evaluation of patella viability after disruption of the arterial circulation. Am J Sports Med 15(5):490-493

26. Shim S, Leung G (1986) Blood supply of the knee joint: a microangiographic study in children and adults. Clin Orthop Relat Res 208:119-125

27. Slater RNS, Spencer JD, Churchill MA, Bridgeman GP, Brookes M (1991) Observations on the intrinsic blood supply to the human patella: disruption correlated with articular surface degeneration. J R Soc Med 84:606-607

28. Swiontkowski MF, Ganz R, Schlegel U, Perren SM (1987) Laser Doppler flowmetry for clinical evaluation of femoral head osteonecrosis. Clin Orthop Relat Res 218:181-185

29. Swiontkowski MF, Tepic S, Perren SM, Moor R, Ganz R, Rahn BA (1986) Laser Doppler flowmetry for bone blood flow: correlation with microsphere estimates and evaluation of the effect of intracapsular pressure on femoral head blood flow. J Orthop Res 4:362-371

30. Terry GC (1989) The anatomy of the extensor mechanism. Clin Sports Med 8(2):163-177

31. Thomas JR, Nelson JK (1996) Research methods in physical activity. Human Kinetics, Champaign 